\title{
Are pressure fluctuation-based equilibrium methods really worse than nonequilibrium methods for calculating viscosities?
}

\author{
Ting Chen, Berend Smit, ${ }^{\text {a) }}$ and Alexis T. Bell ${ }^{\text {a) }}$ \\ Department of Chemical Engineering and Energy Biosciences Institute, University of California, \\ Berkeley, California 94720-1462, USA
}

(Received 19 August 2009; accepted 6 November 2009; published online 30 December 2009)

[doi:10.1063/1.3274802]

Nonequilibrium methods have become increasingly popular for calculating the viscosity of liquids. ${ }^{1-6}$ The use of nonequilibrium methods is frequently rationalized by the notion that pressure fluctuation-based equilibrium methods, such as those using the Green-Kubo (GK) formula and the Einstein relation, do not converge well, and hence are less suitable than nonequilibrium methods. ${ }^{1}$ In this short note, we revisit the systems on which this conclusion is based. We show that a slightly different way of analyzing the data allows us to use equilibrium molecular dynamics methods to compute viscosities with comparable accuracy and reliability as the nonequilibrium techniques considered in Ref. 1 and the reverse nonequilibrium molecular dynamics method. ${ }^{7}$

The shear viscosity can be computed using the GK formula ${ }^{8,9}$

$$
\eta=\frac{V}{10 k_{B} T} \int_{0}^{\infty}\left\langle\sum_{\alpha \beta} P_{\alpha \beta}(0) P_{\alpha \beta}(t)\right\rangle d t
$$

or, equivalently, using the Einstein relation,

$$
\eta=\frac{V}{20 k_{B} T_{t \rightarrow \infty}} \lim _{t} \frac{d}{d t}\left\langle\sum_{\alpha \beta}\left(G_{\alpha \beta}(t)-G_{\alpha \beta}(0)\right)^{2}\right\rangle .
$$

Equation (2) requires computing the mean-square displacement (MSD) of the time integral of the pressure tensor, ${ }^{9}$ where $G_{\alpha \beta}(t)=\int_{0}^{t} P_{\alpha \beta}\left(t^{\prime}\right) d t^{\prime} . P_{\alpha \beta}$ is the symmetrized traceless portion of the stress tensor, $\sigma_{\alpha \beta}$, defined as

$$
P_{\alpha \beta}=\frac{1}{2}\left(\sigma_{\alpha \beta}+\sigma_{\beta \alpha}\right)-\frac{1}{3} \delta_{\alpha \beta}\left(\sum_{y} \sigma_{y y}\right),
$$

where $\delta_{\alpha \beta}$ is the Kronecker delta and $\delta_{\alpha \beta}=0$ when $\alpha \neq \beta$. It should be noted that some researchers ${ }^{10}$ use a weighting factor of $4 / 3$ for the diagonal components $(\alpha=\beta)$ and 1 for the off-diagonal components $(\alpha \neq \beta)$, but here we take the view in Refs. 8 and 9 that all weighting factors should be 1 . We note further that Eqs. (1) and (2) utilize the information of the diagonal components of the pressure tensor to improve statistics. To compare our results with those in Ref. 1, we also compute the viscosity using only the off-diagonal components of the pressure tensor, i.e., when $\alpha \neq \beta$, and then the normalization factors in Eqs. (1) and (2) become 6 and 12, respectively. The overhead in computing the stress tensor in the simulation is very small once the forces have been calculated. The computed pressure-pressure time correlation function was averaged over multiple time origins spaced every ten time steps and block averaging method was used to obtain viscosity estimate and corresponding standard deviation within specified correlation time period.

Molecular dynamics simulations were performed for a 1000-particle Lennard-Jones (LJ) fluid using the LAMMPS (Ref. 11) package in the NVT ensemble with a Nosé-Hoover thermostat. All quantities are expressed in reduced units: $T^{*}=k_{B} T / \varepsilon, \quad \rho^{*}=\rho \sigma^{3}, \quad r^{*}=r / \sigma, \quad t^{*}=t \sqrt{\varepsilon / m \sigma^{2}}, \quad$ and $\quad \eta^{*}$ $=\eta \sigma^{2} / \sqrt{m \varepsilon}$. The simulations were carried out for the same conditions as those used in Ref. $1\left(T^{*}=2.0, \rho^{*}=0.452\right.$, a time step of 0.01 and a cut-off radius of 5). The system was equilibrated for a period of $1 \times 10^{3}$ followed by a production run of $t^{*}=1 \times 10^{5}$.

Figure 1 shows the viscosity curves determined using the fluctuation information of all components and only the offdiagonal components of the pressure tensor [see SI (Ref. 12] for a typical example of a pressure-pressure time correlation function). The inset shows the short correlation time behavior. Since the GK formula and the Einstein relation give identical results, we do not distinguish these two approaches. We find that our viscosity calculation converges quickly and reaches a plateau within a correlation time of 2 . For the inset, the two viscosity averages obtained over a correlation time period between 2 and 6 (or 400 samples) were $0.549 \pm 0.002$ and $0.545 \pm 0.002$ using all components and only offdiagonal components of the pressure tensor, respectively. The corresponding viscosity averages over a long correlation time period of $2-150$ are $0.545 \pm 0.006$ and $0.555 \pm 0.006$.

To compare our results directly with those of Hess, we also performed a simulation of exactly the same duration as reported in Ref. $1\left(t^{*}=5 \times 10^{3}\right.$, which is a factor of 20 times shorter). Of course, the statistics of this short simulation is poorer. An average over a correlation time period of $2-6$ gives a viscosity of $0.549 \pm 0.004$ using all pressure components information and a viscosity of $0.52 \pm 0.05$ using only the off-diagonal pressure components information. These results are consistent with our longer simulation. However, if we use a longer correlation time, we see the same divergence as reported by Hess.

At this point, it is important to mention that one can reliably use the data only at short correlation times to calculate viscosity. Indeed, in agreement with Mondello and Grest, ${ }^{9}$ we found that it is better to make viscosity estimates at early correlation times rather than at later correlation times once the plateau in the GK integral, or equivalently, the linear regime in MSD has been reached, to avoid larger statistical errors. Caution also has to be taken in determining the 


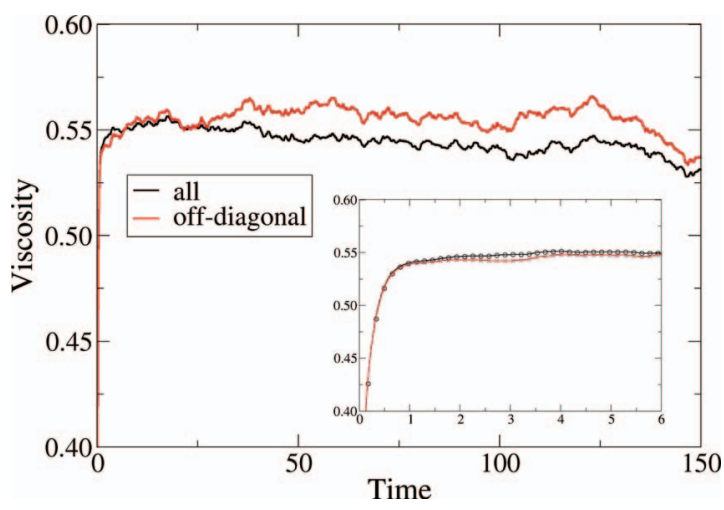

FIG. 1. The viscosity as a function of maximum time taken in the integration of Eq. (1) for the LJ fluid. The viscosity reaches a plateau for an integration time longer than the correlation length. At longer times, the statistical error in the correlation functions causes the deviation from the plateau value. The inset shows the short-time behavior where lines and symbols are results based on the GK formula and the Einstein relation, respectively.

time at which to make an estimate of the viscosity. If this time is too short, a systematic error can be introduced if the plateau in GK formula is not reached or not well defined, or the slope in Einstein MSD plot is not taken in the linear regime. If the time at which the viscosity is evaluated is too long, the integration will be significantly influenced by the noise in the pressure correlation function and as a consequence the viscosity estimates fluctuate heavily leading to unreliable predictions. The optimal simulation time at which to evaluate viscosity depends on the details of the systems.

Our viscosity of 0.549 differs from the one reported in Ref. 1, which ranges between 0.444 and 0.462 . We compared our equilibrium calculations with the reverse nonequilibrium molecular dynamics (RNEMD) (Ref. 7) method. These calculations were performed on a 2197-particle LJ system with a time step of 0.005 and a velocity exchange rate of $\mathrm{W}$ $=100$ in the NVE ensemble. At $T^{*}=2.0$ and $\rho^{*}=0.452$, RNEMD calculation gave a viscosity of $0.543 \pm 0.002$, in excellent agreement with that from the equilibrium approach. Additionally, 12 adjacent state points were simulated. As shown in Fig. 2, we found that our computed viscosities

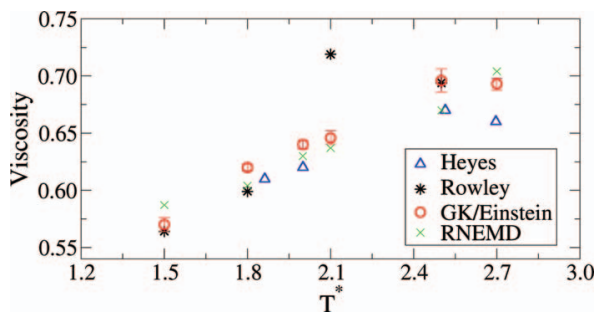

FIG. 2. Viscosities of LJ fluids as a function of temperature at $\rho^{*}=0.5$ [the data for $\rho^{*}=0.4$ can be found in the SI (Ref. 12)]. Triangles and stars are viscosity data from Refs. 14 and 13. Circles and crosses are viscosity estimates obtained using equilibrium molecular dynamics and RNEMD, respectively. obtained using equilibrium approaches and those obtained using the RNEMD method are consistent with each other, and both are in good general agreement with values reported by Rowley and Painter ${ }^{13}$ and Heyes. ${ }^{14}$ All equilibrium simulations in Fig. 2 were run for a period of $5 \times 10^{4}$ and all RNEMD simulations were run for a period of $1 \times 10^{4}$. All viscosities from equilibrium approaches are averages over a correlation time of 2-20. For the LJ fluid at the triple point: $T^{*}=0.722$ and $\rho^{*}=0.8442$, our computed viscosity of $3.269 \pm 0.002$ matches well the most recent and accurate viscosity value reported by Viscardy et al., ${ }^{15}$ $3.291 \pm 0.057$.

To ensure that a similar accuracy can be obtained for more complex fluids, we have extended these calculations to compute the viscosity of SPC and SPC/E water models. The details of these calculations can be found in the SI. ${ }^{12}$ For both LJ fluids and water models, we obtained viscosity data with comparable accuracy and reliability as nonequilibrium methods. Our results also confirm Hess' conclusion that the equilibrium method can result in poorly converged viscosities. However, in this note, we show that these convergence issues can be addressed with enough statistics and by a careful selection of the integration times. For a simple LJ fluid, equilibrium molecular dynamics has computational costs similar to RNEMD method. Equilibrium methods, however, do not require additional adjustments that nonequilibrium methods usually need, such as choosing optimum velocity exchange rate in RNEMD method or appropriate strength of perturbation or proper shear rate, or an extrapolation to obtain zero-shear rate viscosity.

Funding for this work was provided by BP through the Energy Biosciences Institute.

${ }^{a)}$ Authors to whom correspondence should be addressed. Electronic addresses: berend-smit@berkeley.edu and alexbell@berkeley.edu.

${ }^{1}$ B. Hess, J. Chem. Phys. 116, 209 (2002).

${ }^{2}$ V. R. Vasquez, E. A. Macedo, and M. S. Zabaloy, Int. J. Thermophys. 25, 1799 (2004).

${ }^{3}$ J. A. Backer, C. P. Lowe, H. C. J. Hoefsloot, and P. D. Iedema, J. Chem. Phys. 122, 154503 (2005).

${ }^{4}$ Y. J. Wu, H. L. Tepper, and G. A. Voth, J. Chem. Phys. 124, 024503 (2006).

${ }^{5}$ Z. H. Hu and C. J. Margulis, J. Phys. Chem. B 111, 4705 (2007).

${ }^{6}$ J. Picalek and J. Kolafa, Mol. Simul. 35, 685 (2009).

${ }^{7}$ F. Müller-Plathe, Phys. Rev. E 59, 4894 (1999).

${ }^{8}$ P. J. Daivis and D. J. Evans, J. Chem. Phys. 100, 541 (1994).

${ }^{9}$ M. Mondello and G. S. Grest, J. Chem. Phys. 106, 9327 (1997).

${ }^{10}$ O. Borodin, G. D. Smith, and H. Kim, J. Phys. Chem. B 113, 4771 (2009).

${ }^{11}$ S. Plimpton, J. Comput. Phys. 117, 1 (1995).

${ }^{12}$ See EPAPS supplementary material at http://dx.doi.org/10.1063/ 1.3274802 for time correlation example and the viscosity calculation of water.

${ }^{13}$ R. L. Rowley and M. M. Painter, Int. J. Thermophys. 18, 1109 (1997).

${ }^{14}$ D. M. Heyes, Phys. Rev. B 37, 5677 (1988).

${ }^{15}$ S. Viscardy, J. Servantie, and P. Gaspard, J. Chem. Phys. 126, 184512 (2007). 